Bundesgesundheitsbl 2013 $\cdot 56: 1368-1375$ DOI 10.1007/s00103-013-1800-8

Online publiziert: 28. August 2013

๑) Springer-Verlag Berlin Heidelberg 2013
D. Walter ${ }^{1} \cdot$ K. Atzpodien ${ }^{1}$. C. Pins ${ }^{2}$ - O. Wichmann ${ }^{1} \cdot$ S. Reiter ${ }^{1}$

${ }^{1}$ Fachgebiet Impfprävention, Robert Koch-Institut, Berlin

${ }^{2}$ Gesellschaft für Innovative Marktforschung mbH, Heidelberg

\section{Einflussfaktoren auf die Inanspruchnahme von Impfungen durch Jugendliche mit Migrationshintergrund}

\author{
Eine qualitative Untersuchung mit \\ Jugendlichen, Müttern und Ärzten
}

\section{Hintergrund}

Mehr als ein Viertel der in Deutschland lebenden Kinder und Jugendlichen hat einen Migrationshintergrund [1]. Daten zum Impfstatus von Personen mit Migrationshintergrund liegen für Deutschland nur eingeschränkt vor. Schuleingangsuntersuchungen zeigen, dass Kinder mit Migrationshintergrund genauso gut bzw. teilweise - je nach Impfung und Herkunftsland - sogar besser geimpft sind als Kinder ohne Migrationshintergrund [2]. Im Kinder- und Jugendgesundheitssurvey (KiGGS) fanden sich jedoch Impflücken bei Kindern und Jugendlichen mit eigener Migrationserfahrung sowie generell bei Jugendlichen [3].

Neben der Analyse von Impfquoten differenziert nach Herkunftsgruppen ist es als Grundlage für die Gestaltung impfpräventiver Maßnahmen wichtig, Wissen, Einstellung und Verhalten der jeweiligen Zielgruppen vertiefend zu analysieren. Dies gilt nicht zuletzt für die kultursensible Gestaltung dieser Maßnahmen, z. B. wenn dabei in Bildlichkeit oder Formulierung auf die Lebenswelt von Personen mit Migrationshintergrund eingegangen werden soll. Zur Identifizierung möglicher Barrieren und Ressourcen für die Inanspruchnahme von Impfungen durch Jugendliche eignen sich qualitative Metho- den [4]. Moderierte Fokusgruppen ermöglichen einen vertiefenden Einblick in die untersuchte Zielgruppe, insbesondere in frühen Entwicklungsstadien von Studien, in denen Ideen entwickelt, Konzepte erstellt und Einstellungen erfragt werden sollen, die durch quantitative Methoden möglicherweise nicht erfasst werden $[5,6]$. Sie sind daher auch ein etabliertes Instrument, um Barrieren und Ressourcen für präventives Verhalten - wie Handhygiene [7] oder Impfungen - sowohl in der Bevölkerung $[8,9,10,11]$ als auch bei medizinischen Fachkräften zu analysieren $[12,13,14]$. Durch diese Methode können zudem Erkenntnisse über unter Umständen schwer erreichbare Gruppen gewonnen werden, wie z. B. über Personen mit Migrationshintergrund $[15,16]$. Für Impffragestellungen haben sich Fokusgruppen als Methode bewährt, z. B. bei der Impfung gegen humane Papillomviren (HPV) $[17,18]$.

Die präsentierten Fokusgruppengespräche mit Jugendlichen und Müttern von Jugendlichen mit Migrationshintergrund sowie mit niedergelassenen Ärzten ${ }^{1}$ wurden im Rahmen des vom Bundesministerium für Gesundheit geförderten

\footnotetext{
${ }^{1}$ Aus Gründen der Lesbarkeit wird im Text die männliche Form für Ärztinnen und Ärzte verwendet.
}

Forschungsprojekts „Entwicklung einer Strategie zur Steigerung der Impfquoten bei Kindern und Jugendlichen mit Migrationshintergrund“ durchgeführt [4]. Übergeordnetes Ziel der Fokusgruppenuntersuchung war es, mögliche Einflussfaktoren auf die Inanspruchnahme von Impf- und Informationsangeboten sowie die Einstellungen, das Wissen und das Verhalten von Jugendlichen mit Migrationshintergrund in Bezug auf Impfungen aus der Sicht verschiedener Zielgruppen zu ermitteln.

\section{Methoden}

\section{Stichprobengewinnung und Zusammensetzung der Fokusgruppen}

Die Organisation und Durchführung der Fokusgruppen erfolgte durch die Gesellschaft für innovative Marktforschung $\mathrm{mbH}$ (GIM) in enger Kooperation mit dem Fachgebiet „Impfprävention“ des Robert Koch-Instituts.

Insgesamt wurden 72 Teilnehmende für 11 Fokusgruppen rekrutiert. Neben Berlin kamen die Teilnehmenden aus dem Großraum Mannheim, Ludwigshafen und Heidelberg.

Da der primäre Fokus auf Jugendlichen lag, wurden 8 Fokusgruppengesprä- 
Tab. 1 Zusammensetzung der Fokusgruppen mit Jugendlichen, Müttern und Ärzten in den Sample-Points Berlin und Heidelberg

\begin{tabular}{|c|c|c|c|}
\hline \multirow[t]{2}{*}{ Zielgruppen } & \multicolumn{2}{|c|}{ Sample-Points, Durchführung } & \multirow[t]{2}{*}{$\Sigma$} \\
\hline & $\begin{array}{l}\text { Berlin, } \\
\text { 23.-25.03.2011 } \\
\text { Items Teststudio }\end{array}$ & $\begin{array}{l}\text { Heidelberg, } \\
\text { 28. und 29.03.2011 } \\
\text { GIM Heidelberg }\end{array}$ & \\
\hline $\begin{array}{l}\text { Jugendliche mit } \\
\text { türkischem Migra- } \\
\text { tionshintergrund }\end{array}$ & $\begin{array}{l}1 \text { Fokusgruppe mit } \\
\text { Jungen }(6 \mathrm{TN})\end{array}$ & $\begin{array}{l}1 \text { Fokusgruppe mit } \\
\text { Mädchen (6TN) }\end{array}$ & $2 \mathrm{FGDs}(\mathrm{N}=12 \mathrm{TN})$ \\
\hline $\begin{array}{l}\text { Jugendliche mit } \\
\text { arabischem Migra- } \\
\text { tionshintergrund }\end{array}$ & $\begin{array}{l}1 \text { Fokusgruppe mit } \\
\text { Mädchen (6TN) }\end{array}$ & $\begin{array}{l}1 \text { Fokusgruppe mit } \\
\text { Jungen (6TN) }\end{array}$ & $2 \mathrm{FGDs}(\mathrm{N}=12 \mathrm{TN})$ \\
\hline $\begin{array}{l}\text { Jugendliche mit } \\
\text { russischem Migra- } \\
\text { tionshintergrund }\end{array}$ & $\begin{array}{l}1 \text { Fokusgruppe mit } \\
\text { Jungen }(6 \mathrm{TN})\end{array}$ & $\begin{array}{l}1 \text { Fokusgruppe mit } \\
\text { Mädchen (6TN) }\end{array}$ & $2 \mathrm{FGDs}(\mathrm{N}=12 \mathrm{TN})$ \\
\hline $\begin{array}{l}\text { Jugendliche ohne Mi- } \\
\text { grationshintergrund }\end{array}$ & $\begin{array}{l}1 \text { Fokusgruppe mit } \\
\text { Mädchen (6TN) }\end{array}$ & $\begin{array}{l}1 \text { Fokusgruppe mit } \\
\text { Jungen ( } 6 \mathrm{TN})\end{array}$ & $2 \mathrm{FGDs}(\mathrm{N}=12 \mathrm{TN})$ \\
\hline $\begin{array}{l}\text { Mütter von Jugend- } \\
\text { lichen mit türkischem } \\
\text { Migrationshinter- } \\
\text { grund }\end{array}$ & 1 Fokusgruppe (8TN) & - & 2 FGDs $(\mathrm{N}=16 \mathrm{TN})$ \\
\hline $\begin{array}{l}\text { Mütter von Jugend- } \\
\text { lichen mit russischem } \\
\text { Migrationshinter- } \\
\text { grund }\end{array}$ & - & 1 Fokusgruppe (8TN) & \\
\hline Impfende Ärzte & 1 Fokusgruppe (8TN) & - & $1 \mathrm{FGD}(\mathrm{N}=8 \mathrm{TN})$ \\
\hline$\Sigma$ & 11 Fokusgruppen & & \\
\hline
\end{tabular}

che mit jeweils 6 Jugendlichen im Alter von 14 bis 16 Jahren durchgeführt (davon als Vergleichsgruppe 2 mit Jugendlichen ohne Migrationshintergrund). Ergänzend wurden 2 Fokusgruppengespräche mit jeweils 8 Müttern von jugendlichen Kindern mit türkischem und russischem Migrationshintergrund geführt. Fokusgruppengespräche mit Müttern ohne Migrationshintergrund bzw. mit arabischem Hintergrund wurden aus Ressourcengründen nicht durchgeführt. Zusätzlich erfolgte ein Fokusgruppengespräch mit 8 niedergelassenen impfenden Ärzten der Fachrichtungen Kinder- und Jugendmedizin, Allgemeinmedizin und Gynäkologie aus Berliner Stadtteilen mit einem hohen Anteil an Menschen mit Migrationshintergrund (• Tab. 1).

Die Stichprobenziehung bei den Jugendlichen und Müttern wurde im Sinne einer absichtsvollen Stichprobenziehung in Form eines qualitativen Stichprobenplans realisiert [19]. Hauptunterscheidungsmerkmal zwischen den Gruppen war das Vorhandensein eines Migrationshintergrundes. Der Migrationshintergrund sowie weitere vordefinierte Kri- terien wurden mittels eines Screeningfragebogens erfasst. Der Migrationshintergrund wurde als mindestens einseitiger Migrationshintergrund definiert, d. h., mindestens 1 Elternteil musste in einem der vorher definierten Herkunftsländer geboren und nach Deutschland zugewandert sein. Die Auswahl der Teilnehmenden mit Migrationshintergrund konzentrierte sich auf den in Deutschland häufig vertretenen türkischen, arabischen (arabischer Sprachraum) bzw. russischen Migrationshintergrund. Als russisch wurde die Herkunft der Familien aus den ehemaligen Teilrepubliken der UdSSR/ GUS-Staaten verstanden. Die Mehrheit der teilnehmenden Jugendlichen hatte einen zweiseitigen Migrationshintergrund. Kein Jugendlicher war selbst zugewandert. Weitere Kriterien waren das Alter (14 bis 16 Jahre), eine Mindestaufenthaltsdauer in Deutschland von 2 Jahren sowie der aktuelle Lebensmittelpunkt in Deutschland (mindestens 10 Monate im Jahr). Bei weiteren Kriterien, wie z. B. der hauptsächlich im Alltag und zu Hause verwendeten Sprache, dem Zuwanderungsjahr der Familie und der besuchten
Schulform der Jugendlichen, wurde eine möglichst gleichmäßige Verteilung angestrebt.

Der Erstkontakt zu potenziell Teilnehmenden erfolgte über verschiedene $\mathrm{Zu}$ gangswege: der Großteil über Aushänge in Kultur-, Freizeit- und Beratungseinrichtungen sowie im Schneeballverfahren über soziale Netzwerke. Anschließend wurde über den Screeningfragebogen das Vorliegen der Teilnahmekriterien überprüft. Die teilnehmenden Jugendlichen und Mütter erhielten eine Aufwandsentschädigung. Die Ärzte wurden über bei GIM bestehende Datenbanken und Adressbestände kontaktiert und ebenfalls incentiviert. Die Ärzte sollten in etwa gleichmäßig aus den 3 genannten Fachgruppen und aus Berliner Stadtteilen mit hohem Migrantenanteil stammen. $\mathrm{Zu}$ sätzlich sollten sie mindestens 3 Jahre in ihrer Praxis tätig sein und Impfungen aktiv durchführen.

Die Teilnahme an den Fokusgruppen erfolgte nach vorheriger schriftlicher Einverständniserklärung durch die Teilnehmenden bzw. deren Erziehungsberechtigten.

\section{Durchführung der Fokusgruppen}

Die Fokusgruppengespräche wurden vom 23. bis 29.03.2011 in Berlin und Heidelberg (Teilnehmende aus dem Großraum Mannheim, Ludwigshafen und Heidelberg) in professionellen Teststudios realisiert. Von den 11 Fokusgruppen fanden 5 in Heidelberg und 6 in Berlin statt (• Tab. 1). Die Fokusgruppengespräche dauerten $2 \mathrm{~h}$ und wurden von jeweils einem der beiden erfahrenen GIM-Moderatoren geführt. Um den Einfluss verschiedener Moderatoren möglichst gering zu halten und gleichzeitig sensibel auf geschlechtsspezifische oder tabuisierte Themen eingehen zu können, wurden die Fokusgruppengespräche mit Jugendlichen geschlechtergetrennt mit entsprechend weiblicher bzw. männlicher Moderation geführt. Die Moderation erfolgte auf der Basis detaillierter Themenleitfäden. Die Leitfäden dienten entsprechend einer explorativen, qualitativen Gesprächsführung als flexibel handhabbarer Themenrahmen, der sicherstellt, dass die im Vorfeld hypothesengeleitet erstellten For- 
schungsfragen in der Diskussion abgebildet wurden. Der Leitfaden für die Jugendlichen und Mütter umfasste als Überthemen die Einstellungen und Gewohnheiten $\mathrm{zu}$ Gesundheit sowie den Informationsbedarf, die Einstellungen, die Nutzung und die Entscheidungsprozesse in Bezug auf Impfungen. Der Leitfaden für die Ärzte fokussierte auf die Situation im Praxisalltag in Bezug auf Impfungen und auf die Versorgung von Jugendlichen und Personen mit Migrationshintergrund.

\section{Dokumentation und Auswertung}

Alle Fokusgruppengespräche wurden per Audio und Video aufgezeichnet. Von den Audioaufnahmen wurden schriftliche Transkripte angefertigt. Auf Basis der Transkripte wurde die Auswertung als qualitative Inhaltsanalyse durchgeführt [20]. Die Inhaltsanalyse und die Erstellung von Themenclustern wurden durch die Moderatoren mittels Gruppierung und Strukturierung des Datenmaterials durchgeführt.

\section{Ergebnisse}

\section{Gesundheitsvorsorge und Impfen aus Sicht der Jugendlichen}

Die Ergebnisse der Fokusgruppengespräche mit Jugendlichen zeigten, dass bei ihnen Gesundheitsthemen unabhängig vom Migrationshintergrund nur eine nachrangige Rolle spielten. Vorherrschend war bei den Jugendlichen der Glaube an die eigene Gesundheit: „wenn man jung ist, denkt man nicht darüber nach" (Zitat eines Jugendlichen). Gesundheitsthemen werden durch Eltern und Großeltern bzw. Lehrer angesprochen; eine aktive eigenständige Informationssuche zum Thema „Gesundheit" fand in der Altersgruppe der teilnehmenden Jugendlichen kaum statt. Auch in der Peergroup werden Gesundheitsthemen nach Aussagen der Jugendlichen kaum diskutiert. Wenn eigene gesundheitliche Fragen auftauchen, sind vor allem die Eltern, das familiäre Netzwerk und das Internet relevante Informationsquellen. In den Familien der Befragten fungierte meistens die Mutter als Hauptverantwortliche für Gesundheitsfragen. In den Fokusgruppen deutete sich an, dass in

Bundesgesundheitsbl 2013 · 56:1368-1375

c) Springer-Verlag Berlin Heidelberg 2013

\section{Walter · K. Atzpodien · C. Pins · O. Wichmann · S. Reiter Einflussfaktoren auf die Inanspruchnahme von Impfungen durch Jugendliche mit Migrationshintergrund. Eine qualitative Untersuchung mit Jugendlichen, Müttern und Ärzten}

\section{Zusammenfassung}

In der Impfprävention stellen die Kommunikation und der Zugang zu Jugendlichen eine besondere Herausforderung dar. Im März 2011 wurden insgesamt 11 moderierte Fokusgruppen mit Jugendlichen mit und ohne Migrationshintergrund, Müttern mit türkischem und russischem Hintergrund sowie mit niedergelassenen Ärzten zur Ermittlung von Barrieren und Ressourcen für impfpräventive Maßnahmen durchgeführt. In den Fokusgruppen zeigte sich, dass die Inanspruchnahme von Impfungen bei Jugendlichen vor allem über die Informations- und Organisationsprozesse in den jeweiligen Familien sowie den Einfluss der impfenden Ärzte gesteuert wird. Bei einer von Jugendlichen als gering wahrgenommenen eigenen Betroffenheit vom Thema "Impfen" ist der nach- lassende Kontakt zu Ärzten beim Übergang vom Kind zum Jugendlichen besonders bedeutend. Migrationsbedingte Faktoren können zusätzlich auf diese Prozesse einwirken. Neben der generellen Verbesserung des Zugangs von Jugendlichen zu Informationen und niederschwelligen Impfangeboten erscheinen zusätzliche kultursensible Interventionen zur Verbesserung der kommunikativen Schnittstelle zwischen Müttern und impfenden Ärzten Erfolg versprechende Maßnahmen, um die Impfquoten im Jugendalter zu verbessern.

Schlüsselwörter Jugendliche · Fokusgruppe · Migrationshintergrund · Impfprävention · J1

\section{Factors influencing the uptake of vaccines by adolescents with migration background. A qualitative study of adolescents, mothers, and physicians}

\section{Abstract}

One of the special challenges in immunization policy is communication and access to adolescents. To identify barriers and enablers for the immunization of this specific target group, we conducted 11 focus group discussions with adolescents with and without migration background, mothers with Turkish and Russian migration background, and physicians in private practice in March 2011. The results showed that for adolescents, the utilization of immunization was guided by informational and organizational processes in families and by the consulting physician. The low awareness of immunization-related issues that was observed among adolescents is especially challenging, because routine vis- its to the doctor often stop with the transition from pediatric to adult health care. Immigration-related factors may additionally affect the utilization of immunization services. Besides improving access to information and lowering the barriers to immunization for adolescents in general, culturally sensitive interventions may be a promising approach for improving communication between mothers and the immunizing physician and thereby improving vaccination coverage in the adolescent target group.

Keywords

Adolescent · Focus groups · Migration background · Vaccination · J1
Familien mit Migrationshintergrund das familiäre Netzwerk insgesamt eine wichtige Rolle zu spielen scheint.

Obwohl ca. ein Drittel der interviewten Jugendlichen angaben, im Rahmen einer Routineuntersuchung im Jugendalter vom Arzt untersucht worden zu sein, hatte die Jugendgesundheitsuntersuchung (J1) als Begriff bei den interviewten Jugendlichen insgesamt nur einen geringen Bekanntheitsgrad.
Wie bei anderen Gesundheitsaspekten fand auch bei Impfungen - Ausnahme bildete die HPV-Impfung bei Mädchen nur eine geringe Auseinandersetzung mit dem Thema statt, „Impfen wird für mich erst aktuell, wenn ich geimpft werde" (Zitat eines Jugendlichen). Impfungen waren ein ambivalentes, aber tendenziell positiv besetztes Thema für die teilnehmenden Jugendlichen. Es zeigten sich kaum generelle Vorbehalte gegenüber Impfun- 


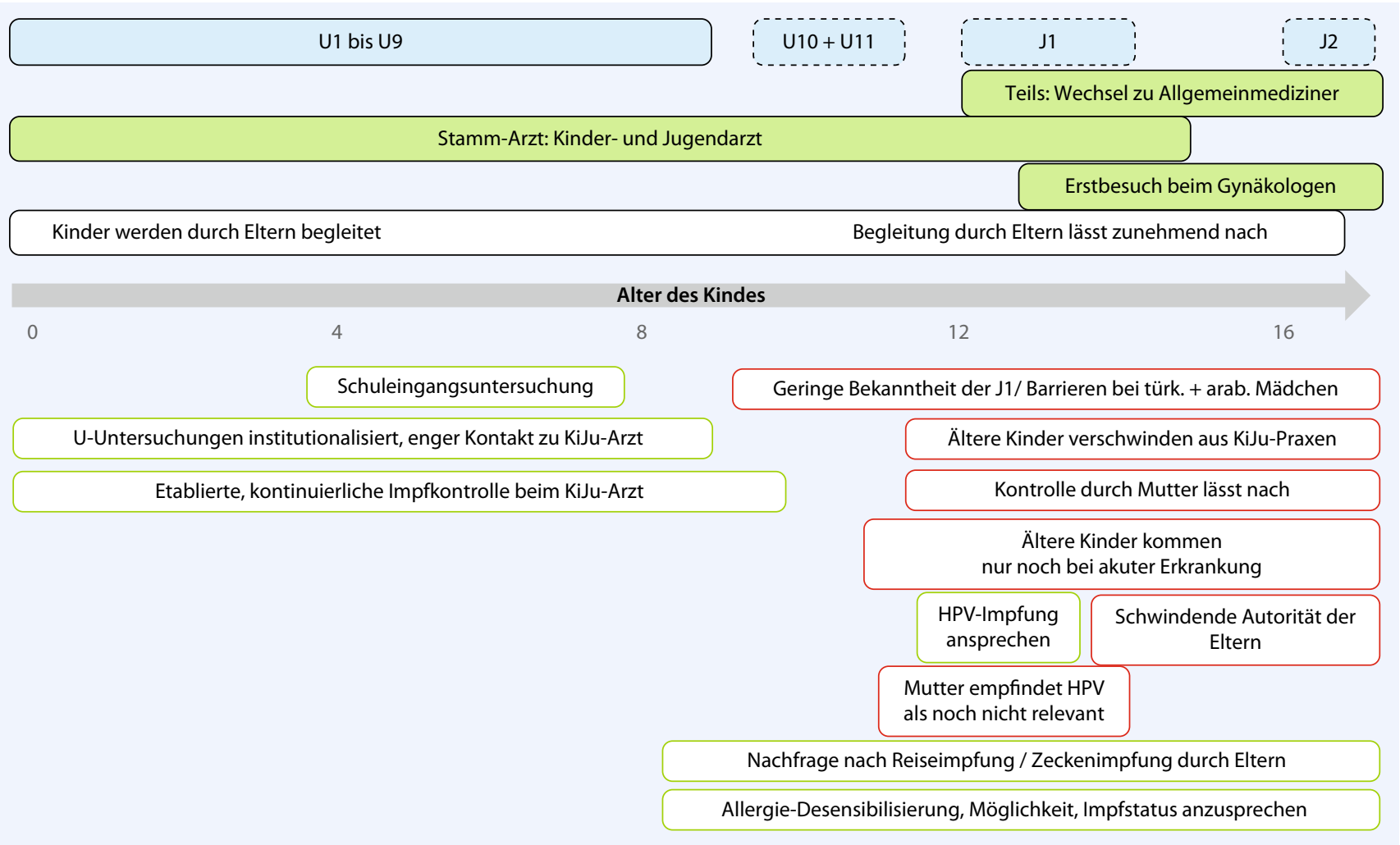

Abb. 1 A Perspektive der teilnehmenden Mütter: Einflussfaktoren im Entwicklungsprozess von Jugendlichen auf Impfentscheidungen (hemmende Faktoren - roter Kasten, fördernde Faktoren - grüner Kasten, Versorgungsverhalten - schwarzer Kasten)

gen. Aktuelle Impfdebatten in den Medien (HPV oder "Schweinegrippe“) wurden jedoch wahrgenommen und führten in diesen Fällen zu einer Zurückhaltung bei der Inanspruchnahme. Geringes Wissen über die Notwendigkeit von Impfungen und Angst vor Nebenwirkungen waren aus Sicht der Jugendlichen hemmende Faktoren für die Inanspruchnahme.

Auffallend war der geringe Kenntnisstand über die Funktionsweise von Impfungen und die Notwendigkeit von Auffrischimpfungen. Sowohl die interviewten Mädchen als auch die russischen Jungen hatten tendenziell ein besseres Wissen über einzelne Impfungen. Aufgrund der geringen eigenen Auseinandersetzung mit dem Thema wurde mehr Information von den meisten Jugendlichen jedoch nicht für wichtig gehalten. Ein aktives Sich-Informieren zum Thema „Impfen" fand bei den teilnehmenden Jugendlichen kaum statt. Das Wissen der Jugendlichen über Impfungen stammte meist von den Eltern (Zitat: „Ich würde immer erst mal meine Mutter fragen"), aus den Medien bzw. vom Arzt.
Bei einer Impfentscheidung ging Initiative und Umsetzung meist von der Mutter aus. Häufigste externe Anlässe zur Vervollständigung von Impfserien waren eine Erinnerung durch den Arzt oder der Arztbesuch aufgrund anderer Anlässe. Als ,interne“ Motivationsgründe wurden neben der Kontrolle durch die Mütter auch Erkrankungen im eigenen Umfeld oder Medienberichte genannt. Bei Mädchen war es auch die eigene Beschäftigung mit der HPV-Impfung. Reisen in die Herkunftsländer wurden hingegen von den Jugendlichen nicht als Anlass gesehen, sich mit Impfungen auseinanderzusetzen. Der Impfpass hatte für die Jugendlichen nur eine geringe Bedeutung und ist etwas, um das sich eher die Eltern und der Arzt kümmern.

Die befragten Jungen befürchteten bei Diskussionen zu Gesundheitsthemen mit Altersgenossen, sich eine Blöße zu geben, während sich die Mädchen mit ihren Freundinnen über sensible Themen austauschen, die sie mit ihren Müttern nicht bereden möchten. Insgesamt zeigten sich in Bezug auf Impfungen keine relevanten geschlechtsspezifischen Unterschiede bei den befragten Jugendlichen. Bei befragten Mädchen bestanden in muslimischen Familien teilweise Vorbehalte gegenüber der HPV-Impfung (Zitat eines Mädchens: „Die denken dann immer gleich, ich will sofort Sex"). Zwischen den interviewten Herkunftsgruppen ergaben sich weder bei den Jugendlichen noch bei den Müttern relevante Unterschiede hinsichtlich des Verhaltens und der grundsätzlichen Einstellungen gegenüber Impfungen. In arabischen und türkischen Familien schien die Tendenz zu bestehen, Jungen früher aus der mütterlichen „Impfkontrolle“ zu entlassen, während in den russischsprachigen Familien ein Verlassen auf externe Autoritäten (Ärzte, Behörden) aufgrund der Impfpraxis in Russland stark ausgeprägt war.

Bei den Zugangswegen zeigten sich innerhalb der interviewten Subgruppen geringfügige Unterschiede. Während bei den Jugendlichen mit türkischem oder arabischem Migrationshintergrund migrationsspezifische Zugangswege und Materialien nur eine geringe Rolle spiel- 


\begin{tabular}{|c|c|c|}
\hline \multicolumn{3}{|c|}{ Anlässe • Impfstatus zu klären • evtl. Impftermin vereinbaren • erfolgte Impfung } \\
\hline Direkter Kontakt zu Jugendlichem & & Kontakt zu Mutter/ Eltern \\
\hline Jugendlicher kommt wegen Verletzung zum Arzt & \multirow{7}{*}{ 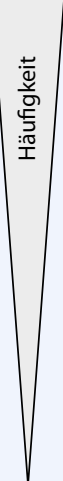 } & Impfungen im Rahmen der U-Untersuchungen \\
\hline & & $\begin{array}{l}\text { Zeckensaison in Presse / Schule thematisiert } \\
\text { Eltern fragen aktiv nach FSME-Impfung }\end{array}$ \\
\hline Beginn der Allergiesaison: Desensibilisierung & & \\
\hline $\begin{array}{l}\text { Jugendlicher kommt zu vereinbartem } \\
\text { Impftermin }\end{array}$ & & $\begin{array}{l}\text { Reiseanlass/ Schulausflug des Kindes } \\
\text { Eltern fragen nach Schutz für sich und/ oder Kind }\end{array}$ \\
\hline Reminder/ Termin z.B. in Impfpass notiert & & \\
\hline \multirow{2}{*}{$\begin{array}{l}\text { Hepatitis/ HPV als Thema in Schule / Unterricht } \\
\text { Aktive Nachfrage seitens der Mädchen } \\
\text { Aber: „normale” Impfungen selten Thema }\end{array}$} & & $\begin{array}{l}\text { Gynäkologe spricht Patientin auf HPV-Impfung } \\
\text { der Tochter an } \\
\text { Beratung der Mutter }\end{array}$ \\
\hline & & $\begin{array}{l}\text { Kinderarzt spricht Mutter aktiv auf Angebot der } \\
\text { J1-Untersuchung an }\end{array}$ \\
\hline
\end{tabular}

Abb. $2<$ Perspektive der teilnehmenden Ärzte: Impfungen bei jugendlichen Patienten - Kontakte, Anlässe (Arzt spricht aktiv an: roter Kasten, Patient spricht aktiv an: grüner Kasten) ten, nutzten russischstämmige Jugendliche auch russischsprachige Medien für die Informationssuche. Trotz der guten deutschen Sprachkenntnisse der interviewten Jugendlichen wurden muttersprachliche Materialien wertgeschätzt.

\section{Impfungen aus Sicht der Mütter}

Im Familienalltag der interviewten türkischen und russischen Mütter spielten Impfungen als Gesprächsthema eine untergeordnete Rolle. Im Vordergrund standen andere gesundheitliche Themen wie Ernährung, Bewegung, Rauchen und Alkohol oder akute Krankheiten. Neben der Kommunikation zu Gesundheitsfragen mit Vertrauten aus dem persönlichen Bekanntenkreis und mit Ärzten wurden von den Befragten zusätzlich Informationsquellen wie Internet, Zeitschriften, Fernsehen oder Broschüren genutzt. Die befragten Mütter fühlen sich in der Regel für die Gesundheit ihrer Kinder verantwortlich. Insbesondere bei älteren Kindern sahen sie jedoch häufig ihren Einfluss schwinden. Auch wenn noch kleinere Geschwister in der Familie waren, gerieten die Jugendlichen manchmal aus dem Blickfeld: „Bei den Kleinen achtet man noch auf alles, bei den Älteren denkt man eher, da ist ja alles in Ordnung" (Zitat einer Mutter).

Bei den teilnehmenden Müttern zeigten sich wie bei den Jugendlichen kaum generelle Vorbehalte gegenüber Impfungen. Fest verankert war das Wissen über die Notwendigkeit der Grundimmuni- sierung bei kleinen Kindern. Bei einem Teil der Mütter herrschte aber Unkenntnis über die empfohlenen Auffrischimpfungen. Die interviewten Mütter waren der Ansicht, dass aktuelle kontroverse Impfdebatten in den Medien, mitunter aufgrund des Bedürfnisses nach klaren Impfempfehlungen, schnell zu Skepsis und Unsicherheit sowie zur Zurückhaltung bei der Inanspruchnahme führen können. Ein stärkerer Austausch mit Ärzten zum Thema „Impfungen“ war zwar gewünscht, fand jedoch selten statt. Bei den befragten Müttern wirkten Sprachdefizite z. T. als Barrieren, um den direkten Kontakt zum Arzt zu suchen. Allerdings wurden Sprach- und Informationsdefizite häufig über einen Rückgriff auf Hilfen in der Familie bzw. im sozialen Netzwerk ausgeglichen. Mütter mit älteren Kindern kommunizierten aktiv deutlich seltener mit anderen Müttern oder mit dem Arzt zum Thema „Impfen“.

Arztwechsel und zunehmende Selbstständigkeit der Kinder gingen einher mit geringer werdender Kontrolle durch die Ärzte und Mütter. Die Wechsel vom Pädiater zum Allgemeinmediziner fanden häufig ab einem Alter von 11 bis 14 Jahren statt. Die Mütter glaubten, dass Teenager sich häufig beim Kinderarzt deplatziert fühlen: „denen ist das mit 13 schon peinlich, zum Kinderarzt zu gehen" (Zitat einer Mutter). $\bullet$ Abb. 1 fasst fördernde (grün) und hemmende (rot) Faktoren in Bezug auf Impfungen bei Kindern und Jugendlichen aus Sicht der Mütter zusammen.
Es bestand unter den teilnehmenden Müttern ein Bedürfnis nach mehr praktischer Unterstützung (Erinnerungen, Argumentationshilfen, Information) sowie nach aktiver Initiierung von Impfungen und möglichst eindeutigen Impfempfehlungen durch externe Autoritäten (Arzt, Schule, Ämter). Solange der Arzt die Kontrolle übernimmt, haben die interviewten Mütter meist ein allgemeines Gefühl der Sicherheit bezüglich des Impfstatus der Kinder. Mit zunehmendem Alter der Kinder verloren Vorsorgeund Impftermine sowohl als Besuchsanlass als auch bei der Kommunikation mit dem Arzt an Relevanz. Gleichzeitig erwarteten die interviewten Mütter neben Information und Aufklärung eine klare ärztliche Empfehlung und äußerten das Bedürfnis nach Entlastung von der Impfentscheidung. Vor allem russischsprachige Mütter merkten an, dass sie aufgrund ihrer eigenen Impfhistorie mehr Klarheit seitens der Ärzte gewohnt sind.

Zweisprachige Materialien hatten für russische und nach Angaben der Jugendlichen auch für arabische Mütter eine höhere praktische Relevanz als für die interviewten türkischen Mütter. Alle Mütter nutzten muttersprachliche Medienangebote. Während soziale Netzwerke bei türkischen Müttern einen hohen Stellenwert hatten, spielte die Betreuung durch russische Ärzte bei den russischsprachigen Müttern eine große Rolle. 


\section{Impfungen bei Jugendlichen aus Sicht der Ärzte}

Die interviewten niedergelassenen Ärzte sahen in der Bevölkerung insgesamt eine eher passive bis kritische Haltung gegenüber der Impfthematik, die ihr aktives Handeln zur Sicherstellung eines ausreichenden Impfschutzes erfordert. Die Initiative zum Impfen und zur Impfkontrolle geht meist vom Arzt aus und erforderte aktive Überzeugungs- und Aufklärungsarbeit. Patienten fragen aktiv eher Impfungen nach, die aus Sicht der befragten Ärzte u. U. weniger wichtig sind (z. B. gegen FSME), hingegen werden wichtige Impfungen eher nachlässig behandelt (gegen Masern, Röteln, Meningokokken). Nach Meinung der Ärzte erhöht sich bei einer Konkretisierung des Gefährdungspotenzials das Interesse an Impfungen drastisch; „wenn irgendwas passiert, sind die Praxen für 2 Tage voll“ (Zitat eines Arztes). Diese Angst führt aber wohl nur begrenzt $\mathrm{zu}$ einem nachhaltig aktiven Impfverhalten.

Aus Sicht der Befragten sind Unterschiede im Gesundheits- und Vorsorgeverhalten bei Patienten mit Migrationshintergrund stark abhängig vom Integrationsgrad (im Sinne von Kenntnis und Teilhabe am Gesundheitssystem). „Je besser integriert diese Patienten sind, desto mehr ähnelt das Gesundheits- und Impfverhalten (auch in späterem Alter) dem der ,Deutschen.“ (Zitat eines Arztes) Unterschiede im Gesundheitsverhalten wurden eher mit sozialem Status bzw. Bildungsgrad als mit dem Migrationsstatus in Verbindung gebracht. Sprachkenntnis und Aufenthaltsdauer wurden als zentrale Faktoren für die Einbindung in das deutsche Gesundheits- und Vorsorgesystem genannt.

Der Impfstatus und die Impfdokumentation von Kindern mit Migrationshintergrund sind aus Sicht der interviewten Ärzte tendenziell besser. Die Qualität der Impfdokumentation (Existenz eines Impfbuchs und dessen Pflege) ist nach Ansicht der teilnehmenden Ärzte eher vom sozialen Status als vom Migrationshintergrund abhängig. Ihrer Meinung nach besteht bei Patienten mit Migrationshintergrund eine geringere Impfskepsis. Die Impfkontinuität lässt im späteren Kindesalter jedoch oft nach, und Impfungen werden dann nur noch bei konkretem Bedrohungsgefühl (Infektionsfall in sozialem Umfeld, Todesfälle in den Medien) nachgefragt.

Die teilnehmenden Ärzte registrierten bei Jugendlichen eine Abnahme der Arztbesuche, bei Jugendlichen mit Migrationshintergrund teils sogar einen markanten Bruch im Konsultationsverhalten: „Mit 12 sind sie auf einmal weg“ (Zitat eines Pädiaters). Anlässe für Arztbesuche sind dann zunehmend nur noch akute Erkrankungen, bei denen u. U. nicht geimpft werden kann.

Vorsorge- und Impfthemen spielen nach Ansicht der Ärzte als Gesprächsthema mit den Jugendlichen selbst keine große Rolle und werden vorwiegend mit der Mutter besprochen. Mit zunehmendem Alter werden die Jugendlichen jedoch nicht mehr von ihren Müttern begleitet. In $\bullet$ Abb. 2 sind mögliche von den Teilnehmenden genannte Anlässe zusammengefasst, bei denen beim Arztkontakt Impfthemen angesprochen werden könnten. Überwiegend erfolgt die Thematisierung des Impfschutzes von Jugendlichen durch die interviewten Ärzte. Mütter fragen teils aktiv nach, dann aber eher anlassbezogen. Die Inanspruchnahme der J1 führt aus Sicht der Ärzte zu höheren Impfquoten. Diese ist aber wenig bekannt und muss aktiv vom Arzt angesprochen werden. Türkische und arabische Mädchen nehmen die J1-Untersuchung nach Aussagen der teilnehmenden Ärzte nur wenig in Anspruch.

Erfolgreiche Maßnahmen zur Erhöhung der Impfakzeptanz sind für die teilnehmenden Ärzte ein direktes Ansprechen der Eltern und die systematische Erinnerung sowie zielgerichtete Maßnahmen anderer Akteure wie Pädagogen, Schulärzte und öffentliche Einrichtungen. Herstellerfinanzierte Projekte wurden eher kritisch gesehen und die Information durch neutrale Institutionen befürwortet. Vorstellbar waren sowohl Hilfen für den Praxisalltag (mehrsprachige Informationsmaterialien) als auch übergeordnete pharmaunabhängige Informationskampagnen, die die Arbeit der Ärzte unterstützen.

\section{Diskussion}

In Studien deutet sich an, dass Kinder und Jugendliche mit Migrationshintergrund in Deutschland nicht per se schlechter geimpft sind [2, 4]. Nichtsdestotrotz sind die Impfquoten bei Jugendlichen nicht zuletzt auch bei solchen mit Migrationshintergrund verbesserungswürdig [3]. Daten zur Masernimpfung zeigen eine geringere Impfquote für zugewanderte Kinder im Vergleich zu in Deutschland geborenen Kindern [21]. Ziel der vorliegenden Studie war es, die spezifische Situation von Jugendlichen mit Migrationshintergrund in Bezug auf Impfungen näher zu analysieren.

Anhand der Fokusgruppen konnte gezeigt werden, dass die Inanspruchnahme von Impfungen bei Jugendlichen stark durch die Informations- und Organisationsprozesse in den jeweiligen Familien sowie durch die impfenden Ärzte beeinflusst wird. Da das Thema „Impfen“ bei Jugendlichen selbst nur eine geringe Relevanz besitzt, ist die Rolle der Eltern und Ärzte im Impfprozess umso entscheidender. Als Hauptbarriere konnte - unabhängig vom Migrationsstatus - der Wegfall von Strukturen identifiziert werden, der mit dem Übergang vom Kindes- in das Erwachsenenalter einhergeht: sowohl ein Nachlassen der elterlichen Zuständigkeit in Gesundheitsfragen als auch der Übergang vom Kinder- zum Hausarzt. In den Fokusgruppengesprächen bestätigte sich die Rolle von Kinder- und Jugendärzten als zentrale Ansprechpartner für die Gesundheit und damit auch als etablierter Zugangsweg für Impfungen [22]. Der beschriebene Wechsel sowie die mit dem Alter zunehmende Tendenz, nur noch bei akuten Erkrankungen einen Arzt aufzusuchen (im Vergleich zu Kleinkindern, die mit den Eltern regelmäßig zu Vorsorgeuntersuchungen gehen), scheinen einen wichtigen Bruch bei der Inanspruchnahme von Impfungen darzustellen. Bei Mädchen könnte der Besuch eines Gynäkologen ein „Wiedereinstieg“ für die Auseinandersetzung mit Impfungen sein (vor allem Impfung gegen HPV).

Migrationsbedingte Faktoren, wie z. B. der kulturelle oder religiöse Hintergrund, erscheinen auf Basis der Fokusgruppengespräche zur Erklärung von möglichen 
Barrieren und Ressourcen für Impfungen bei Jugendlichen allein nicht ausreichend, sondern wirken eher zusätzlich beeinflussend. Die in der vorliegenden Studie dargestellten Befunde sind daher nicht nur für Jugendliche mit Migrationshintergrund, sondern generell für Jugendliche in Deutschland relevant. Hilfreiche Ressourcen zeigten sich bei Jugendlichen mit Migrationshintergrund in deren Familien in Form einer geäußerten hohen allgemeinen Wertschätzung von Impfungen sowie der Nutzung von sozialen Netzwerken zur Kommunikation über Gesundheitsthemen. Eine Erhöhung der Inanspruchnahme von Vorsorgeuntersuchungen wie der J1 vor allem auch bei Jugendlichen mit Migrationshintergrund [23, 24] scheint ein möglicher Anknüpfungspunkt zur Verbesserung der Impfquoten in dieser Altersgruppe zu sein.

Bei der Impfprävention von Jugendlichen sollte ein Ansatz zugrunde liegen, der Mütter in ihrer Rolle als Verantwortliche für die Gesundheit der Familie unterstützt. Die Mütter sind mithilfe von Ärzten die wichtigste Schnittstelle zwischen den Jugendlichen und impfpräventiven Maßnahmen. Die wichtige Rolle der Mutter für die Impfentscheidung bei jugendlichen Mädchen - vor allem auch für die Impfung gegen HPV - ist in qualitativen Studien gut belegt $[25,26]$. Interventionen sollten Mütter zum einen praktisch unterstützen (z. B. klare Impfempfehlungen, für Mütter mit Migrationshintergrund zusätzlich durch mehrsprachige, kultursensible Informationsangebote). Zum anderen sollten sie Mütter auch befähigen, ihren Einfluss auf das Impfen über das klassische Kindesalter hinaus aufrechtzuerhalten, z. B. beim Übergang zum Allgemeinarzt auf die bestehende Notwendigkeit von Auffrischimpfungen hinzuweisen und bei Besuchsanlässen der Jugendlichen die Mütter über anstehende Impfungen zu informieren.

Aufgrund des geringen Bewusstseins über die Notwendigkeit von Impfungen ist es für die gezielte Ansprache von Jugendlichen u. U. von Vorteil, das Thema „Impfprävention“ mit Gesundheitsthemen zu kombinieren, die in dieser $\mathrm{Al}$ tersgruppe ein höheres Aufmerksamkeitspotenzial haben. Interventionen bei Jugendlichen sollten zielgruppenspezifisch und kultursensibel gestaltet sein, um eine hohe Akzeptanz sicherzustellen. Mehrsprachige Materialien sind jedoch möglicherweise eher wichtig, wenn Eltern in einer Intervention angesprochen werden. Aufgrund der oben beschriebenen Übergangsproblematik sollten weitere Akteure stärker eingebunden werden: Sowohl die Schule als auch der öffentliche Gesundheitsdienst sollten Jugendliche vermehrt über Impfungen informieren und zu diesen motivieren [27].

Insbesondere komplexe Problemlagen und durch multiple Einflussfaktoren bestimmtes Verhalten können mittels standardisierter Erhebungen (z. B. mittels Fragebögen) oft nur bedingt oder unvollständig untersucht werden [28]. Fokusgruppen erlauben als qualitative Methode einen vertiefenden Einblick in solche Forschungsfragen, die Generalisierbarkeit ihrer Ergebnisse muss aber sorgfältig abgewogen werden. Die in der Studie identifizierten Ergebnisse erscheinen jedoch plausibel und wurden von allen 3 Gruppen reflektiert. Es wurden allerdings nur Personen mit türkischem, arabischem und russischem Migrationshintergrund in der Studie eingeschlossen. Dies sind zudem keine homogenen Gruppen, wie sich beispielhaft an den unterschiedlichen Migrationsgeschichten russischsprachiger Personen und deren Auswirkung auf die Gesundheit zeigt [29]. Präventives Verhalten wird nicht zuletzt stark von sozioökonomischen Rahmenbedingungen, der persönlichen Migrationsgeschichte oder der Aufenthaltsdauer beeinflusst [30]. In der vorliegenden Studie wurden keine Mütter ohne Migrationshintergrund und keine selbst zugewanderten Jugendlichen befragt. Insbesondere in der letzten Gruppe zeigten sich geringere Impfquoten in quantitativen Studien, sodass nicht auszuschließen ist, dass in dieser Gruppe migrationsspezifische Barrieren stärker zum Tragen kommen [2, 21].

\section{Fazit}

Auf Basis der Fokusgruppen kann festgestellt werden, dass die Aufmerksamkeit für Impfungen bei Jugendlichen generell verbessert werden sollte. Obwohl migrationsbedingte Faktoren in den untersuchten Gruppen nicht allein aus- schlaggebend zu sein scheinen, können die spezifischen Ressourcen für zielgerichtete Interventionen in der Impfprävention genutzt werden. Erfolg versprechend scheint z. B. die Nutzung spezifischer Informationsstrukturen in den $\mathrm{Mi}$ grantengruppen (z. B. soziale Netzwerke). Ärzte sollten als zentrale Ansprechpartner für Impfungen mit zielgruppenspezifischen, herstellerunabhängigen Maßnahmen und Materialien für die Arzt-Patienten-Kommunikation unterstützt werden. Vor allem Hausärzte sollten sensibilisiert werden, Impfthemen bei Besuchen von Jugendlichen aufgrund akuter Beschwerden anzusprechen. Nicht nur die Inanspruchnahme der J1 sollte verbessert, sondern jeder Arztkontakt sollte genutzt werden, um den Impfstatus zu überprüfen. Bei Mädchen bietet die HPV-Impfung einen guten Anknüpfungspunkt, um das Impfthema aufzugreifen. Insbesondere bei der Kommunikation mit der Elterngeneration können möglicherweise kultursensible Ansätze, wie z. B. auch zweisprachige Materialien, die Inanspruchnahme von Impfangeboten zusätzlich verbessern.

\section{Korrespondenzadresse}

\section{Walter}

Fachgebiet Impfprävention,

Robert Koch-Institut

DGZ-Ring 1, 13086 Berlin

walterph@web.de

Danksagung. Wir danken den Teilnehmerinnen und Teilnehmern sowie der Moderatorin und dem Moderator der Fokusgruppen. Das Projekt wurde finanziert durch das Bundesministerium für Gesundheit.

Interessenkonflikt. D. Walter, K. Atzpodien, C. Pins, $O$. Wichmann und $S$. Reiter geben an, dass kein Interessenkonflikt besteht.

Dieser Beitrag beinhaltet keine Studien an Menschen oder Tieren. 


\section{Literatur}

1. Statistisches Bundesamt (2011) Aktueller Mikrozensus 2010. In: Statistisches Bundesamt (Hrsg) Fachserie 1 Reihe 2.2, Migration in Deutschland 2010

2. Pfaff G (2011) Impfprävention bei Kindern und Jugendlichen mit Migrationshintergrund. In: Nationale Impfkonferenz 2011 „Impfen - Wirklichkeit und Visionen". Ministerium fpr Arbeit und Sozialordnung, Familien, Frauen und Senioren - Baden Württemberg. Stuttgart, S 66-73

3. Pöthko-Müller C, Kuhnert R, Schlaud M (2007) Durchimpfung und Determinanten des Impfstatus in Deutschland. Ergebnisse des Kinder- und Jugendgesundheitssurveys (KiGGS). Bundesgesundheitsbl Gesundheitsforsch Gesundheitsschutz 50:851-862

4. Atzpodien K (2011) Bericht zum Expertenworkshop „Impfprävention bei Kindern und Jugendlichen mit Migrationshintergrund". Epid Bull 48:435-438

5. Barbour RS (2005) Making sense of focus groups. Med Educ 39:742-750

6. Krueger R, Casey M (2009) Focus groups. A practical guide for applied research. Sage, Los Angeles

7. Meilicke G, Weissenborn A, Biederbick W, Bartels $C$ (2008) Mit Wasser und Seife gegen die Grippe. Das Handewaschen als Infektionsschutz-Empfehlung fur die Bevolkerung - 7 Hypothesen aus einer qualitativen Studie zu Hygiene, Grippe und Pandemie. Bundesgesundheitsblatt Gesundheitsforschung Gesundheitsschutz 51:1273-1279

8. Bhat-Schelbert K, Lin CJ, Matambanadzo A et al (2012) Barriers to and facilitators of child influenza vaccine - perspectives from parents, teens, marketing and healthcare professionals. Vaccine 30:2448-2452

9. Leask J, Chapman S, Hawe P, Burgess M (2006) What maintains parental support for vaccination when challenged by anti-vaccination messages? A qualitative study. Vaccine 30:7238-7245

10. Mills E, Jadad AR, Ross C, Wilson K (2005) Systematic review of qualitative studies exploring parental beliefs and attitudes toward childhood vaccination identifies common barriers to vaccination. J Clin Epidemiol 58:1081-1088

11. Hilton S, Petticrew M, Hunt K (2007) Parents' champions vs. vested interests: who do parents believe about MMR? A qualitative study. BMC Public Health 7:42

12. Draper $\mathrm{H}$, Wilson S, Ives J et al (2008) Healthcare workers' attitudes towards working during pandemic influenza: a multi method study. BMC Public Health 8:192

13. Ives J, Greenfield S, Parry JM et al (2009) Healthcare workers' attitudes to working during pandemic influenza: a qualitative study. BMC Public Health 9:56

14. Aghaizu A, Elam G, Ncube F et al (2011) Preventing the next,SARS' - European healthcare workers' attitudes towards monitoring their health for the surveillance of newly emerging infections: qualitative study. BMC Public Health 11:541

15. Mixer RE, Jamrozik K, Newsom D (2007) Ethnicity as a correlate of the uptake of the first dose of mumps, measles and rubella vaccine. J Epidemiol Community Health 61:797-801

16. Dingoyan D, Schulz H, Mosko M (2012) The willingness to participate in health research studies of individuals with Turkish migration backgrounds: barriers and resources. Eur Psychiatry 27(Suppl 2):S4S9
17. Scarinci IC, Garces-Palacio IC, Partridge EE (2007) An examination of acceptability of HPV vaccination among African American women and Latina immigrants. JWomens Health (Larchmt) 16:12241233

18. Louis-Nance TR, Flournoy MW, Clinton KS et al (2012) The females against cancer educational series: a qualitative evaluation of mother/daughter knowledge and perceptions of human papillomavirus and its related cancers. J Natl Med Assoc 104:194-198

19. Schreier M (2007) Qualitative Stichprobenkonzepte. In: Naderer G, Balzer E (Hrsg) Qualitative Marktforschung in Theorie und Praxis. Wiesbaden, S 232-245

20. Mayring $P$ (2003) Qualitative Inhaltsanalyse. Grundlagen und Techniken Beltz UTB., Weinheim

21. Pöthko-Müller C, Ellert U, Kuhnert R et al (2009) Vaccination coverage against measles in Germanborn and foreign-born children and identification of unvaccinated subgroups in Germany. Vaccine 27:2563-2569

22. Zylka-Menhorn V (2011) Impfen im Kindesalter: Zentraler Kommunikationspartner ist der Arzt. Dtsch Ärztebl 108:A-1104/B-1912/C-1912

23. Hagen B, Strauch S (2011) The J1 adolescent health check-up: analysis of data from the German KiGGS survey. Dtsch Arztebl Int 108:180-186

24. Zeeb H, Baune B, Vollmer W et al (2004) Health situation of and health service provided for adult migrants - a survey conducted during school admittance examinations. Gesundheitswesen 66:7684

25. Klötzler A, Kolip P (2012) Entscheidungsfindung zur HPV-Impfung - Eine qualitative Studie mit jugendlichen Mädchen. Gesundheitswesen 74:716 721

26. Gowda C, Schaffer SE, Dombkowski KJ, Dempsey AF (2012) Understanding attitudes toward adolescent vaccination and the decision-making dynamic among adolescents, parents and providers. BMC Public Health 12:509

27. Elsäßer G (2008) Jugendimpfung - Herausforderung und Chancen. Kinderarztl Prax 79:31-35

28. Peterson-Sweeney K (2005) The use of focus groups in pediatric and adolescent research. J Pediatr Health Care 19:104-110

29. Tselmin S, Korenblum W, Reimann M et al (2007) The health status of Russian-speaking immigrants in Germany. Horm Metab Res 39:858-861

30. Spallek J, Zeeb H, Razum O (2010) Prevention among immigrants: the example of Germany. BMC Public Health 10:92

\section{Neue Empfehlungen der Ständigen Impf- kommission veröffentlicht}

Die STIKO, die Ständige Impfkommission am Robert Koch-Institut, hat im Epidemiologischen Bulletin 34/2013 den neuen Impfkalender veröffentlicht. Hinzugekommen ist die Empfehlung für eine Rotavirus-Schutzimpfung bei Säuglingen. Veränderungen gibt es auch bei den Empfehlungen zur Hepatitis-B- und zur Influenza-Impfung. Die neu empfohlene Rotavirus-Impfung wird als Schluckimpfung gegeben, die Impfserie sollte im Alter von 6 bis 12 Wochen beginnen und je nach Impfstoff bis zur vollendeten 24. oder 32. Lebenswoche beendet sein. Eine englischsprachige Veröffentlichung zu den wissenschaftlichen Grundlagen der Empfehlung zur Rotavirus-Impfung ist in der Juli-Ausgabe des Bundesgesundheitsblatts bereits erschienen, die deutschsprachige Begründung erscheint im Epidemiologischen Bulletin 35/2013. Bei der Hepatitis B-Impfung ist nach einer erfolgreich durchgeführten Grundimmunisierung im Allgemeinen keine Auffrischimpfung notwendig. Wie bisher wird die Kontrolle des Impferfolgs empfohlen. Die STIKO hat außerdem die in der bisherigen Hepatitis B-Impfempfehlung aufgeführten 8 Indikationsgruppen in 3 Indikationsgruppen zusammengefasst. Bei der Influenza-Impfung empfiehlt die STIKO, bei Kindern im Alter von 2 bis 6 Jahren, bei denen wegen einer Grundkrankheit eine Impfung empfohlen ist, bevorzugt einen Impfstoff zu verwenden, der in die Nase gesprüht wird. Damit erhofft sich die STIKO eine höhere Akzeptanz der jährlich zu wiederholenden Influenza-Impfung. Außerdem wird die Influenza-Impfung jetzt auch Personen empfohlen, die eine geimpfte Risikoperson betreuen. Grund ist, dass die Influenza-Impfung keinen 100\%igen Schutz bietet. Das gilt insbesondere für ältere oder immungeschwächte Menschen, die somit auch bei Impfung indirekt von einem Impfschutz der sie betreuenden Personen profitieren.

Weitere Informationen unter: www.stiko.de und www.rki.de/impfen

Quelle:

Robert Koch-Institut, Berlin, www.rki.de 